\title{
Comparação das resistências ao embutimento paralelo às fibras de madeiras de pínus oocarpa, cumaru e pínus taeda
}

\author{
Comparison of the embedment strength parallel to the \\ grain of woods of pinus oocarpa, cumaru and pinus taeda
}

Diego Henrique de Almeida Pontifícia Universidade Católica de Minas Gerais Poços de Caldas - MG - Brasil

Ricardo de Mello Scaliante Universidade de São Paulo São Carlos - SP - Brasil

André Luis Christoforo Universidade Federal de São Carlos São Carlos - SP - Brasil

Francisco Antônio Rocco Lahr Universidade de São Paulo São Carlos - SP - Brasil

Julio Cesar Molina Universidade Estadual Paulista Itapeva - SP - Brasil

Carlito Calil Junior Universidade de São Paulo São Carlos - SP - Brasil

Recebido em 28/05/13 Aceito em 19/06/14

\section{Diego Henrique de Almeida \\ Ricardo de Mello Scaliante \\ André Luis Christoforo \\ Francisco Antonio Rocco Lahr \\ Julio Cesar Molina \\ Carlito Calil Junior}

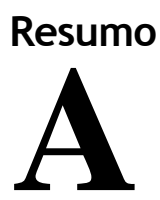

norma brasileira NBR 7190 (ABNT, 1997) estabelece as diretrizes para a determinação da resistência da madeira ao embutimento com pinos metálicos por meio de ensaios laboratoriais ou, na ausência deles, disponibiliza equações que permitem estimar a resistência da madeira ao embutimento. A referida norma admite ainda que na direção paralela às fibras da madeira a resistência à compressão seja igual à resistência ao embutimento. O objetivo deste trabalho consistiu em analisar as resistências de embutimento e de compressão, ambos na direção paralela às fibras, utilizando madeiras das espécies pínus oocarpa (Pinus oocarpa Shiede), cumaru (Dipterix odorata) e pínus taeda (Pinus taeda L.). Para comparação das resistências foi utilizado o teste estatístico de hipótese conhecido como Teste T. Os resultados da análise estatística revelaram a não equivalência entre os valores de resistência apenas para as madeiras de Pinus oocarpa, evidenciando, assim, a importância da determinação da resistência da madeira ao embutimento através de ensaios laboratoriais normalizados.

Palavras-chave: Madeira. Resistência. Embutimento. Compressão. Estruturas.

\section{Abstract}

The Brazilian standard NBR 7190-1997 provides guidelines for embedment strength determination on timber structures for metal dowels fastenings using laboratory tests or, in their absence, prescribes procedures to estimate the embedment strength. This standard assumes that the embedment strength parallel to the grain can be considered to be equivalent to the compressive strength parallel to the grain. The aim of this research work is to analyse the embedment strength parallel to grain and the compression strength parallel to grain for Pinus oocarpa (Pinus oocarpa Shiede), cumaru (Dipterix odorata) and pínus taeda (Pinus taeda L.) wood species. For comparing the strengths, the hypothesis the statistical T test was used. The results indicated that there is no equivalence between the strength values only for the pinus oocarpa wood specie, showing the importance of determining the embedding strength through standard laboratory tests.

Keywords: Wood. Strength. Embedment. Compression. Timber structures. 


\section{Introdução}

A necessidade de ligação entre elementos estruturais para a construção de estruturas de madeira implica estudos referentes à resposta da madeira quanto às solicitações impostas e aos tipos de ligações utilizadas entre os elementos estruturais.

A ligação entre elementos estruturais de madeira mais difundida é a que utiliza pinos metálicos, como, por exemplo, os pregos, os parafusos e, mais recentemente, os segmentos de barras de aço comum, pois eles possuem baixo custo de aquisição e praticidade para utilização em diversas configurações de ligações.

A norma "Projeto de Estruturas de Madeira" (ABNT, 1997), apresenta as premissas para a realização do dimensionamento de ligações com pinos metálicos.Esse documento normativo associa dois fenômenos que configuram a ruptura de uma ligação: o embutimento do pino metálico na madeira e a flexão do pino metálico, fazendo-se necessário, neste caso, o conhecimento das propriedades físicas e mecânicas da madeira utilizada e a resistência ao escoamento do aço empregado na confecção do pino metálico.

Alguns fatores podem influenciar a resistência da madeira ao embutimento. Podem ser citados o efeito da pré-furação, o espaçamento entre os pinos e as distâncias entre borda da madeira e centro do pino, direção das fibras, ângulo entre a direção de atuação da carga e as fibras da madeira, espécie de madeira e o diâmetro do pino metálico (ALMEIDA, 1987; VALLE, 1999; OLIVEIRA, 2001; STAMATO; CALIL JUNIOR, 2001; ALMEIDA, 2014).

Almeida (1987), em pesquisa a respeito das ligações com pregos, inicialmente considerou a resistência da madeira à compressão paralela às fibras como um bom parâmetro para estimar a resistência da ligação. Porém, após a realização de ensaios laboratoriais, observou o embutimento do prego na parede do furo, tendo surgido, então, no Brasil, o primeiro estudo brasileiro referente à resistência da madeira ao embutimento. $\mathrm{O}$ mesmo autor define a resistência da madeira ao embutimento como a resistência à ruptura localizada da madeira pela pressão do pino metálico contra a parede do furo. No entanto, o primeiro estudo realizado no mundo sobre o embutimento da madeira foi realizado por Johansen (1949).

A NBR 7190 (ABNT, 1997), em seu Anexo B, intitulado "Determinação das propriedades das madeiras para projetos de estruturas", fornece as diretrizes para a estimativa da resistência da madeira ao embutimento com pinos metálicos na direção paralela às fibras $\left(f_{\mathrm{e} 0}\right)$, que é dada, neste caso, pela razão entre a força que causa a deformação específica residual de $2 \%\left(\mathrm{~F}_{\mathrm{e} 0}\right)$ e a área diametral da parede do furo (A), sendo a área definida como o produto entre a espessura da peça de madeira (t) e o diâmetro do pino metálico (d) (Equação 1).

$\mathrm{f}_{\mathrm{e} 0}=\frac{\mathrm{F}_{\mathrm{e} 0}}{\mathrm{~A}}=\frac{\mathrm{F}_{\mathrm{e} 0}}{\mathrm{t} . \mathrm{d}}$

Eq. 1

$\mathrm{Na}$ ausência de ensaios laboratoriais, a norma brasileira adota a resistência da madeira ao embutimento $\left(f_{e, 0}\right)$ na direção paralela às fibras como sendo igual à resistência à compressão na mesma direção $\left(\mathrm{f}_{\mathrm{c} 0}\right)$.

A força que causa a deformação específica residual de $2 \%$ é determinada no diagrama força versus deformação específica. Porém, o que ocorre é o deslocamento do pino metálico, e não a deformação específica do corpo de prova como um todo, assim como ocorre no ensaio de resistência na compressão paralela às fibras.

A norma europeia EN 383 (EUROPEAN..., 2007) considera um deslocamento máximo de $5 \mathrm{~mm}$ do pino metálico para caracterizar o embutimento do pino metálico na madeira, e a norma americana ASTM D5764 (AMERICAN..., 2007) considera o embutimento do pino metálico através de um método gráfico que leva em consideração um deslocamento residual do pino em relação ao corpo de prova igual a $5 \%$ de seu diâmetro.

Oliveira (2001) disserta sobre o fato de a norma brasileira igualar os valores das duas resistências, que acarreta na diminuição da resistência do pino metálico, o que influencia no número de conectores utilizados na ligação.

Logsdon, Finger e Rosa (2001) realizaram a caracterização mínima e simplificada da madeira da espécie Cambará (Vochisya guianensis Aubl.) e utilizaram as relações preconizadas pela norma brasileira para determinação das resistências das referidas madeiras ao embutimento nas direções paralela e normal às fibras, devido à necessidade de pesquisas a fim de melhorar as relações fornecidas pela NBR 7190 (ABNT, 1997) para a caracterização simplificada.

Almeida (2011) determinou valores médios de resistência ao embutimento e de compressão nas direções paralela, inclinada a $45^{\circ}$ e normal em relação às fibras de madeiras de pínus taeda (Pinus taeda) e Eucalipto citriodora (Corymbia citriodora), encontrando valores distintos entre 
essas duas propriedades em todas as combinações (inclinação das fibras-espécie de madeira) estudadas.

Nascimento et al. (2012) determinaram os valores médios de resistência ao embutimento paralelo às fibras da madeira das espécies Corymbia citriodora, Eucalyptus paniculata e Eucalyptus pellita, sendo estas iguais a 51,00 $\mathrm{MPa}, 82,10 \mathrm{MPa}$ e 49,20 MParespectivamente, e, nessa mesma ordem, também foram determinados os valores médios de resistência à compressão paralela às fibras, sendo estes iguais a $65 \mathrm{MPa}, 78,50 \mathrm{MPa}$ e 63,20 MPa. Pelas diferenças encontradas, os autores chamaram a atenção para que seja revista pela NBR 7190 (ABNT, 1997) a relação que estabelece a equivalência entre a resistência ao embutimento e a resistência na compressão paralela às fibras da madeira.

Este trabalho teve o objetivo de calcular e comparar as resistências ao embutimento com pino metálico e de compressão, ambos na direção paralela às fibras, em madeiras provenientes de espécies nativas e exóticas brasileiras, de acordo as recomendações da NBR 7190 (ABNT, 1997).

\section{Material e métodos}

Para o desenvolvimento desta pesquisa foram realizados ensaios laboratoriais utilizando madeiras de uma espécie nativa e de duas exóticas brasileiras, provenientes de lotes considerados homogêneos,com teor de umidade na ordem de $12 \%$. As madeiras utilizadas foram pínus oocarpa (Pinus oocarpa Shiede), pínus taeda (Pinus taeda L.) e cumaru (Dipterix odorata), sendo as duas primeiras coníferas, e a última, dicotiledônea. Foram confeccionados 6 corpos de prova para a realização dos ensaios de resistência ao embutimento, e 6 para os ensaios de resistência à compressão, na direção paralela em relação às fibras, para as três espécies estudadas, considerando as dimensões padronizadas pela NBR 7190 (ABNT, 1997).

Os corpos de prova de embutimento na direção paralela às fibras foram ensaiados na máquina universal de ensaios mecânicos DARTEC, com capacidade de $100 \mathrm{kN}$, utilizando pinos metálicos com $1 \mathrm{~cm}$ de diâmetro, tendo estes resistência ao escoamento $\left(\mathrm{f}_{\mathrm{yk}}\right)$ igual a $240 \mathrm{MPa}$ (Figura 1). Para a realização dos ensaios de compressão paralela às fibras foi utilizada a máquina universal de ensaios mecânicos AMSLER, que possui capacidade de $250 \mathrm{kN}$ (Figura 2). Todos os ensaios foram realizados de acordo com as recomendações propostas no Apêndice B da NBR 7190 (ABNT, 1997).

A verificação da equivalência estatística entre as resistências à compressão e ao embutimento paralelo às fibras das madeiras investigadas foi realizada mediante o emprego de teste de hipótese (Teste $\mathrm{t}$ ), com o auxílio do software Minitab ${ }^{\circledR}$ versão 14. O Teste t foi avaliado para um nível de significância $(\alpha)$ de 5\%, estipulando-se a equivalência entre médias para a hipótese nula $\left(\mathrm{H}_{0}\right.$ : $\left.\mu_{1}=\mu_{2}\right)$ e a não equivalência como a hipótese alternativa $\left(\mathrm{H}_{0}: \mu_{1} \neq \mu_{2}\right)$. Dessa forma, um P-valor superior ao nível de significância implica se aceitar $\mathrm{H}_{0}$, rejeitando-a em caso contrário.

Para validar os resultados do Teste $\mathrm{t}$ (intervalo de confiança entre as médias), foi necessário verificar se as variáveis-respostas confrontadas apresentaram distribuição normal. Para tanto, foi utilizado o teste de Anderson-Darling, com nível de significância de 5\%, consistindo em uma distribuição normal como hipótese nula e não normalidade nas distribuições como hipótese alternativa. Acusada a não normalidade na distribuição para alguma das propriedades mecânicas investigadas, foi utilizada a Transformada de Johnson, utilizando-se dos dados normalizados na realização dos testes de hipótese.

\section{Resultados e discussão}

A Tabela 1 apresenta os resultados obtidos das resistências à compressão e de embutimento na direção paralela às fibras para as três espécies de madeira investigadas, sendo $\mathrm{x}_{\mathrm{m}}$ a média amostral, e $\mathrm{Cv}$, o coeficiente de variação. 
Figura 1 - Corpos de prova instrumentados para a realização dos ensaios de resistência ao embutimento na direção das fibras

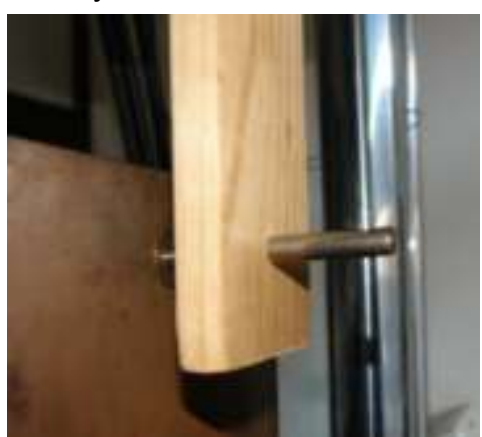

(a) Pínus oocarpa

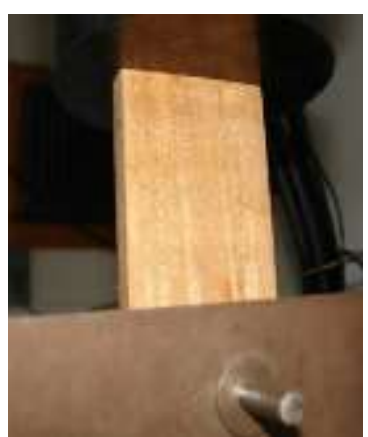

(b) Cumaru

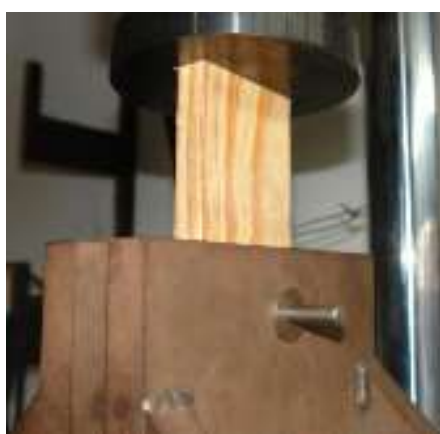

(c) Pínus taeda

Figura 2 - Corpos de prova instrumentados para a realização dos ensaios de resistência à compressão paralela às fibras

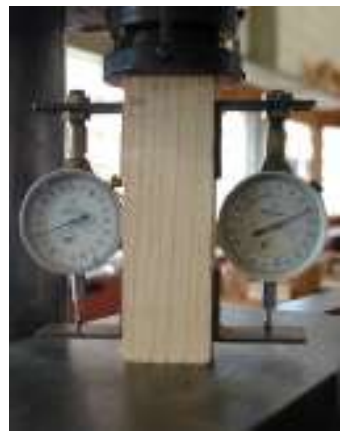

(a) Cumaru



(b) Pínus taeda

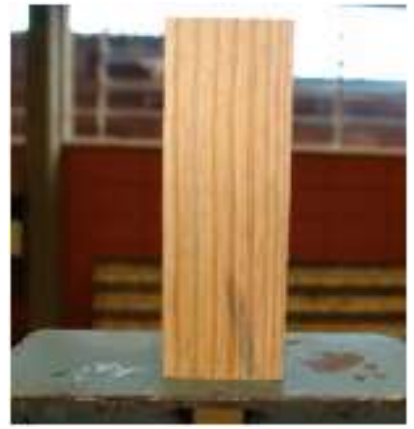

(c) Pínus oocarpa

Tabela 1 - Resultados das resistências da madeira ao embutimento e à compressão paralela às fibras das espécies das madeiras estudadas

\begin{tabular}{ccccccc}
\hline \multirow{2}{*}{$\mathbf{C P}$} & \multicolumn{2}{c}{ Pínus oocarpa } & \multicolumn{2}{c}{ Cumaru } & \multicolumn{2}{c}{ Pínus taeda } \\
\cline { 2 - 7 } & $\mathbf{f}_{\mathbf{c}, \mathbf{0}}(\mathbf{M P a})$ & $\mathbf{f}_{\mathbf{e}, \mathbf{0}}(\mathbf{M P a})$ & $\mathbf{f}_{\mathbf{c}, \mathbf{0}}(\mathbf{M P a})$ & $\mathbf{f}_{\mathbf{e}, \mathbf{0}}(\mathbf{M P a})$ & $\mathbf{f}_{\mathbf{c}, \mathbf{0}}(\mathbf{M P a})$ & $\mathbf{f}_{\mathbf{e}, \mathbf{0}}(\mathbf{M P a})$ \\
\hline 1 & 33,40 & 12,00 & 98,27 & 84,00 & 24,89 & 23,00 \\
2 & 31,84 & 9,50 & 98,70 & 82,00 & 27,73 & 23,00 \\
3 & 30,83 & 14,50 & 99,78 & 63,00 & 22,58 & 19,50 \\
4 & 33,54 & 10,00 & 67,22 & 70,00 & 29,95 & 30,50 \\
5 & 37,09 & 10,00 & 61,61 & 64,00 & 30,26 & 20,80 \\
6 & 40,35 & 7,20 & 68,60 & 80,00 & 29,15 & 26,00 \\
\hline $\mathrm{X}_{\mathrm{m}}$ & 34,51 & 10,53 & 82,36 & 73,83 & 27,43 & 23,8 \\
$\mathrm{Cv}$ & 10 & 24 & 22 & 14 & 11 & 17 \\
\hline
\end{tabular}

A Figura 3 ilustra os resultados do teste de normalidade de Anderson-Darling para as resistências na compressão e de embutimento na direção paralela às fibras das madeiras. Com exceção da resistência à compressão paralela da madeira de cumaru (Figura 3c), as demais variáveis-respostas apresentaram normalidade em suas distribuições, sendo isso observado pelos $\mathrm{P}$ valores encontrados, ambos superiores ao nível de significância do teste.

A Figura 4 ilustra o resultado da Transformada de Johnson para a resistência à compressão paralela da madeira de cumaru, cuja transformada é apresentada pela Equação 2, sendo $\mathrm{X}$ a variável (independente) dos dados originais, e Y, a variável (dependente) dos dados transformados. De maneira a possibilitar o emprego do Teste $t$ na verificação de equivalência estatística entre a resistência à compressão paralela e à resistência ao embutimento paralelo da madeira de cumaru, a Equação 2 foi também empregada na transformação dos dados da resistência ao embutimento na direção paralela às fibras da madeira.

$Y=-0,225706+0,253847 \cdot \log \left(\frac{X-61,5762}{99,7857-X}\right) \quad$ Eq. 2 
Figura 3 - Resultados dos testes de normalidade de Anderson-Darling

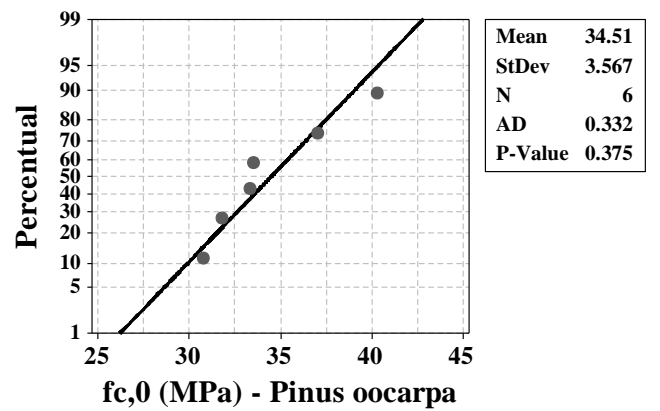

(a)

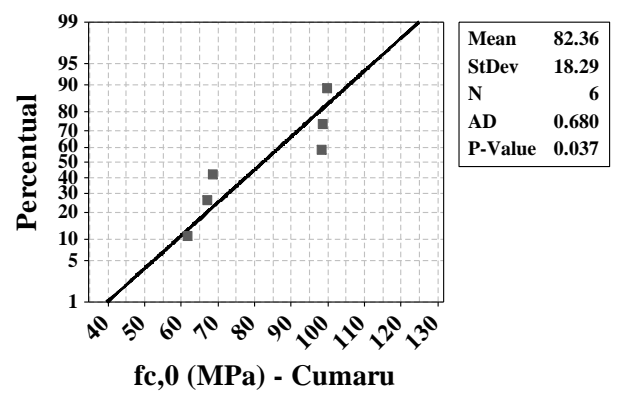

(c)

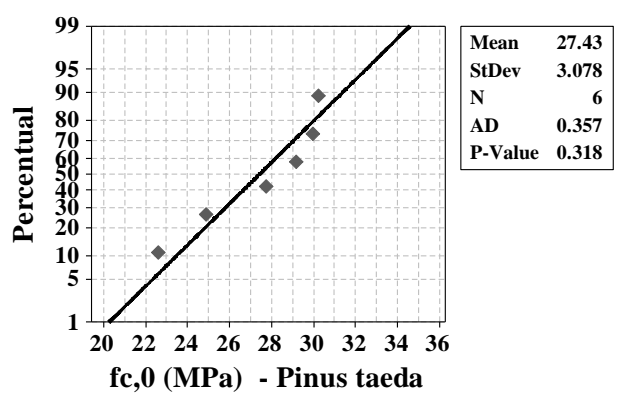

(e)

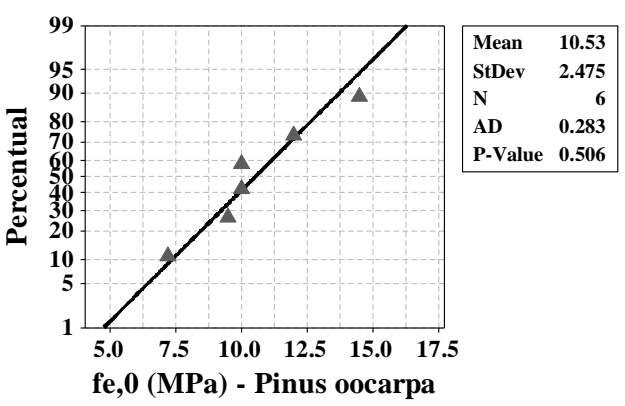

(b)

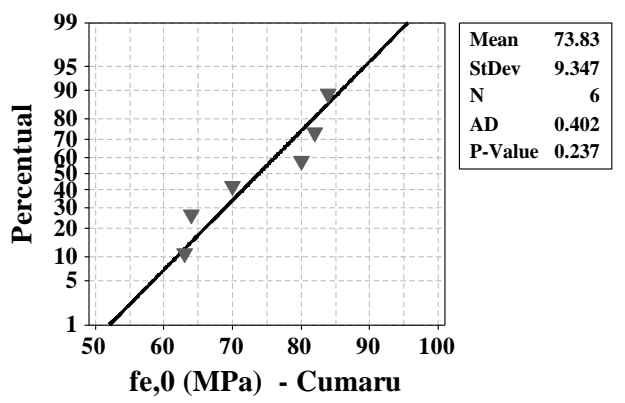

(d)

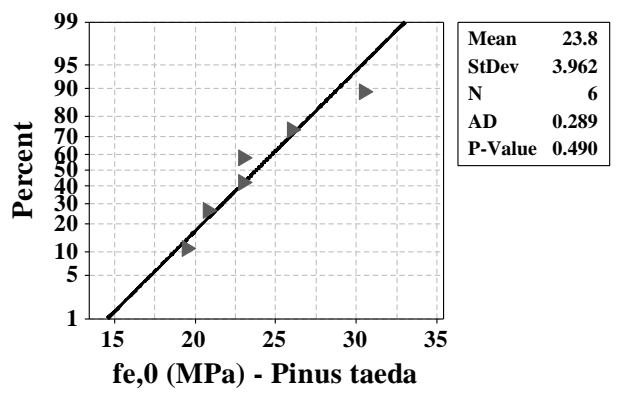

(f)

Figura 4 - Transformada de Johnson para a resistência à compressão paralela às fibras da madeira de cumaru

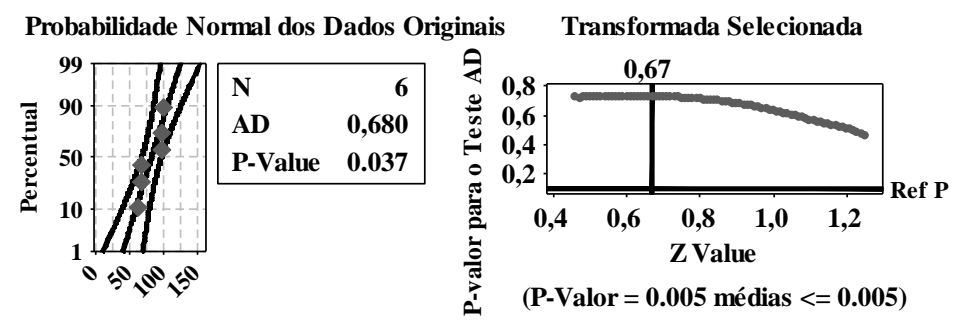

Probabilidade Normal dos Dados transformados

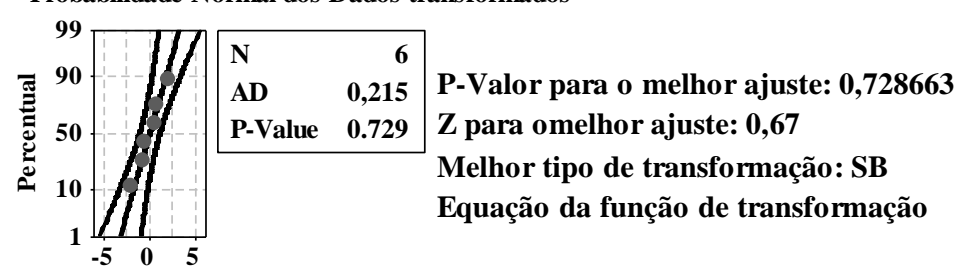


Figura 5 - Resultados do teste de normalidade de Anderson-Darling para os dados transformados: resistência na direção paralela às fibras da madeira de cumaru

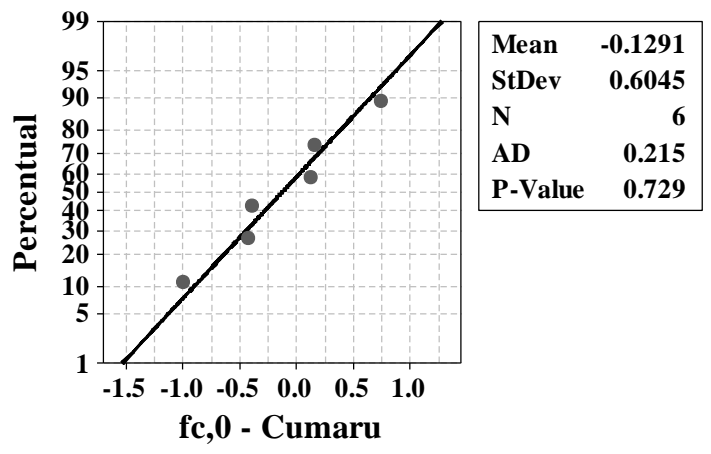

(a) Compressão

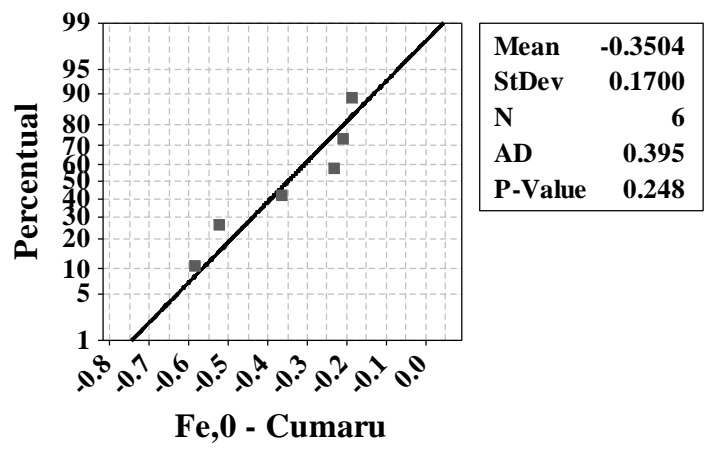

(b) Embutimento

Tabela 2 - Resultados do teste de hipótese

\begin{tabular}{ccccc}
\hline Espécies & & Graus de Liberdade & IC $(\boldsymbol{\mu})$ & P-valor \\
\hline Pínus oocarpa & $\mathrm{f}_{\mathrm{c}, 0}$ & 8 & $19,89 \leq \mu \leq 29,06$ & 0,000 \\
& $\mathrm{f}_{\mathrm{e}, 0}$ & 5 & $-0,438 \leq \mu \leq 0,880$ & 0,427 \\
\hline Cumaru & $\begin{array}{l}\mathrm{f}_{\mathrm{c}, 0} \\
\mathrm{f}_{\mathrm{e}, 0}\end{array}$ & 5 & $-1,01 \leq \mu \leq 8,26$ & 0,110 \\
\hline Pínus taeda & $\begin{array}{l}\mathrm{f}_{\mathrm{c}, 0} \\
\mathrm{f}_{\mathrm{e}, 0}\end{array}$ & 9 & & \\
\hline
\end{tabular}

A Figura 5 ilustra os resultados do teste de normalidade de Anderson-Darling dos dados (resistências) transformados da madeira de cumaru, sendo constatada a normalidade nas distribuições pelos P-valores encontrados, ambos superiores ao nível de significância do teste, validando o emprego do Teste $t$.

A Tabela 2 apresenta os intervalos de confiança (IC) e os resultados do teste de hipótese referente à verificação da equivalência estatística entre a resistência à compressão paralela e ao embutimento das espécies de madeira aqui avaliadas. A relação de equivalência entre as resistências $\left(\mathrm{f}_{\mathrm{c}, 0}=\mathrm{f}_{\mathrm{e}, 0}\right)$ estipulada pela NBR 7190 (ABNT, 1997) não foi válida apenas para a madeira da espécie Pínus oocarpa, por apresentar $\mathrm{P}$-valor inferior ao nível de significância do teste.

\section{Conclusão}

Os resultados apontaram para que a relação entre as resistências à compressão e ao embutimento na direção paralela às fibras das madeiras, segundo a NBR 7190 (ABNT, 1997), deve ser realizada para as classes de resistência, visto a não equivalência encontrada para a madeira de pínus oocarpa. Novas pesquisas na mesma temática devem ser realizadas com o intuito de investigar um maior número de espécies de madeira, possibilitando estabelecer conclusões gerais referentes ao embutimento dessa matéria-prima.

\section{Referências}

ALMEIDA, D. H. Proposta de Método de Ensaio Para Determinação da Resistência da Madeira ao Embutimento. 130 f. São Paulo, 2014. Dissertação (Mestrado em Engenharia de Estruturas) - Escola de Engenharia de São Carlos, Universidade de São Paulo, São Carlos, 2014.

ALMEIDA, D. H. Análise das Resistências ao Embutimento e de Compressão para Madeiras de Pinus e Eucalipto. 100 f. Itapeva, 2011.

Monografia (Graduação em Engenharia Industrial Madeireira) - Campus Experimental de Itapeva, Universidade Estadual Paulista, Itapeva, 2011.

\section{ALMEIDA, P. A. O. Uniões Pregadas de}

Madeira. 174 f. São Paulo, 1987. Dissertação

(Mestrado em Engenharia de Estruturas) - Escola Politécnica, Universidade de São Paul, São Paulo, 1987.

\section{AMERICAN SOCIETY FOR TESTING AND}

MATERIALS.ASTM D-5764: evaluating dowelbearing strength of wood and wood-based products. Philadelphia, 2007.

ASSOCIAÇÃO BRASILEIRA DE NORMAS TÉCNICAS. NBR 7190: projeto de estruturas de madeira. Rio de Janeiro, 1997. 


\section{EUROPEAN COMMITEE FOR}

STANDARDZATION. EN 383: determination of embedding strength and foundation values for dowel type fasteners. Brussels, 2007.

JOHANSEN, K. W. Theory of Timber Connections. In: International Association for Bridge and Structural Engeneering. Zurick, 1949. v. 9, p. 249-262.

LOGSDON, N. B.; FINGER, Z.; ROSA, L. M. Caracterização da Madeira de Cambará, Vochisya guianensis Aubl. Revista Engenharia Civil, Guimarães, v. 18, p. 49-76, 2001

NASCIMENTO, A. M. et al. Resistências à Compressão e ao Embutimento e Densidade das Madeiras de Corymbia citriodora, Eucalyptus pellita e Eucalyptus paniculata. In: ENCONTRO BRASILEIRO DE MADEIRAS E EM ESTRUTURAS DE MADEIRA, 13., Vitória. 2012. Anais... Vitória: Ibramem, 2012.
OLIVEIRA, M. A. M. Ligações Com Pinos

Metálicos em Estruturas de Madeira.137 f. São

Carlos, 2001. Dissertação (Mestrado em

Engenharia de Estruturas) - Escola de Engenharia de São Carlos, Universidade de São Paulo, São Carlos, 2001.

STAMATO, G. C.; CALIL JUNIOR, C. Resistência ao Embutimento da Madeira Compensada. Cadernos de Engenharia de Estruturas, São Carlos, v. 18, p. 49-76, 2001.

VALLE, A. Rigidez de Ligações Com Parafusos em Estruturas de Madeira Laminada Colada.193 f. 1999. Tese (Doutorado em Engenharia Civil) - Escola Politécnica, Universidade de São Paulo, São Paulo, 1999.

Diego Henrique de Almeida

Pontifícia Universidade Católica de Minas Gerais | Departamento de Engenharia Civil | Av. Padre Francis Cletus Cox, 1661, Cowtry Club | Poços de Caldas - MG - Brasil | CEP 37701-355 | Tel.: (35) 3729-9200 | E-mail.: almeida@pucpcaldas.com.br

Ricardo de Mello Scaliante

Escola de Engenharia de São Carlos | Universidade de São Paulo | Av. Trabalhador Sãocarlense, 400, Centro | São Carlos - SP - Brasil | CEP 13566-590 | Tel.: (016) 3373-8206 | E-mail.: scaliante@usp.br

André Luis Christoforo

Departamento de Engenharia Civil | Universidade Federal de São Carlos | Rodovia Washington Luís, km 235, SP-310 | São Carlos - SP Brasil | CEP 36307-352 | Tel.: (16) 98259-6211 | E-mail.: alchristoforo@yahoo.com.br

\section{Francisco Antônio Rocco Lahr}

Escola de Engenharia de São Carlos | Universidade de São Paulo | Av. Trabalhador Sãocarlense, 400, Centro | São Carlos - SP - Brasil | CEP 13566-590 | Tel.: (016) 3373-8206 | E-mail.: frocco@sc.usp.br

\section{Julio Cesar Molina}

Universidade Estadual Paulista | Rua Geraldo Alckimin, 519, Nossa Senhora de Fátima | Itapeva - SP - Brasil | CEP 13560-970 | Tel.: (15) 3524-9100 | E-mail: molina@itapeva.unesp.br

Carlito Calil Junior

Escola de Engenharia de São Carlos | Universidade de São Paulo | E-mail.: E-mail.: calil@sc.usp.br

Revista Ambiente Construído

Associação Nacional de Tecnologia do Ambiente Construído

Av. Osvaldo Aranha, $99-3^{\circ}$ andar, Centro

Porto Alegre - RS - Brasil CEP $90035-190$

Telefone: +55 (51) 3308-4084

Fax: +55 (51) 3308-4054

www.seer.ufrgs.br/ambienteconstruido

E-mail: ambienteconstruido@ufrgs.br 